\title{
Title: Methane Conversion to Fuels and Chemicals: Opportunities and Approaches
}

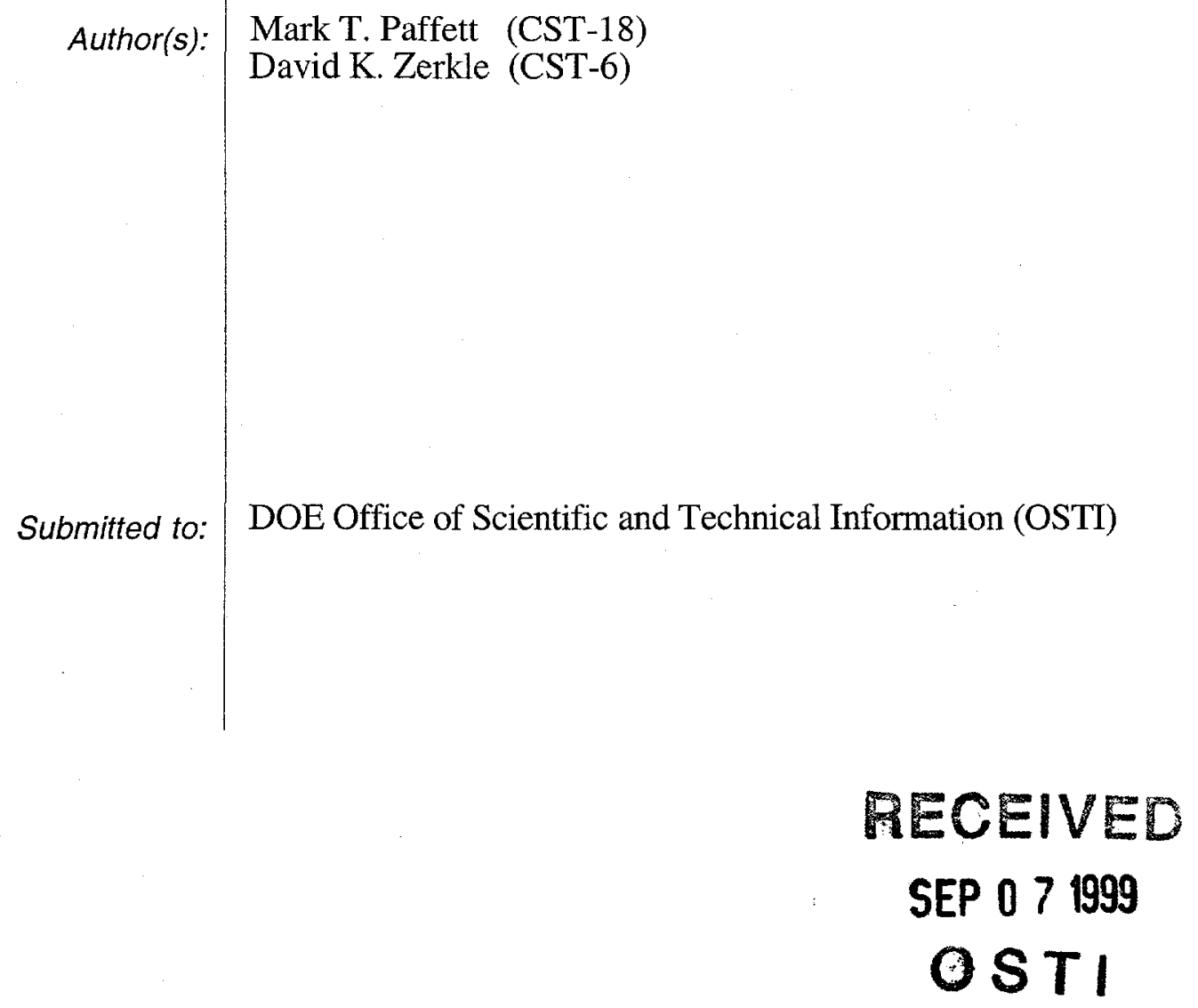

\section{Los Alamos}

NATIONAL LABORATORY

Los Alamos National Laboratory, an affirmative action/equal opportunity employer, is operated by the University of California for the U.S. Department of Energy under contract W-7405-ENG-36. By acceptance of this article, the publisher recognizes that the U.S. Government retains a nonexclusive, royaltyfree license to publish or reproduce the published form of this contribution, or to allow others to do so, for U.S. Government purposes. Los Alamos National Laboratory requests that the publisher identify this article as work performed under the auspices of the U.S. Department of Energy. Los Alamos National Laboratory strongly supports academic freedom and a researcher's right to publish; as an institution, however, the Laboratory does not endorse the viewpoint of a publication or guarantee its technical correctness. 


\section{DISCLAIMER}

This report was prepared as an account of work sponsored by an agency of the United States Government. Neither the United States Government nor any agency thereof, nor any of their employees, make any warranty, express or implied, or assumes any legal liability or responsibility for the accuracy, completeness, or usefulness of any information, apparatus, product, or process disclosed, or represents that its use would not infringe privately owned rights. Reference herein to any specific commercial product, process, or service by trade name, trademark, manufacturer, or otherwise does not necessarily constitute or imply its endorsement, recommendation, or favoring by the United States Government or any agency thereof. The views and opinions of authors expressed herein do not necessarily state or reflect those of the United States Government or any agency thereof. 


\section{DISCLAIMER}

Portions of this document may be illegible in electronic image products. Images are produced from the best available original document. 


\title{
Methane Conversion to Fuels and Chemicals: Opportunities and Approaches
}

\author{
Mark T. Paffett * (CST-18) \\ David K. Zerkle (CST-6)
}

\begin{abstract}
This is the final report of a one-year, Laboratory-Directed Research and Development (LDRD) project at the Los Alamos National Laboratory (LANL). Methane, the primary component of natural gas, has reserves that are on the order of those of petroleum. Processes that utilize these vast supplies of methane will need to be developed to replace dwindling supplies of petroleum in the future. Processes utilizing natural gas promise to be more environmentally friendly, as natural gas as a feedstock is freer of contaminants and more readily purified than petroleum. Short contact time reactor configurations are likely candidates for this application. Our objectives are to develop reactor designs and computer models appropriate for short contact time applications. We have succeeded in assembling both an experimental facility for investigating the performance of short contact time reactors, and a computer simulation that includes full mass and heat transport as well as coupled surface and gas phase detailed chemical kinetics.
\end{abstract}

\section{Background and Research Objectives}

In this project we intended to combine experimentation with theory and computer modeling to produce the fundamental understanding needed to develop and optimize short contact time chemical reactors for gas liquefaction. Huge economic rewards for the U. S. can be realized by the efficient conversion of natural gas to higher hydrocarbons, oxygenates, and olefins. The challenge for gas liquefaction is to oxidize the light organic gases without generating large quantities of water and carbon dioxide. Short contact time reactors operate at high catalyst surface temperatures $\left(800\right.$ to $\left.900^{\circ} \mathrm{C}\right)$ and short reaction times $\left(10^{-5}\right.$ to $\left.10^{-3} \mathrm{sec}\right)$. These conditions produce high surface reaction rates that lead to high conversion, while minimizing non-selective homogenous gas phase reactions.

Although the development and understanding of these types of catalytic reactor systems is in its infancy, several reactor configurations have already been proposed and tested. The short contact time reactor process as described by Schmidt et al. [4-7] for the conversion of light alkanes to fuels and chemicals is a very promising candidate to compete

\footnotetext{
*Principal Investigator, e-mail: mtp@lanl.gov
} 
with conventional steam pyrolysis routes. Improvements in selectivity to synthesis gas and alkene would provide further impetus for practice of this technology on a commercial scale. Our objective is a fundamental understanding of the fluid dynamics and chemical reaction mechanisms driving the observed global conversions and selectivity for short contact time reactors and the opportunity for process improvement. We endeavor to determine, through experimentation, modeling, and collaborations with other members of the team, how operational variables (flow rates, temperature) and design variables (catalyst geometry) affect reactor performance. This information will be compared to the results of various fluid dynamics code calculations that can be used to screen new reactor concepts, to optimize new reactor designs, and identify important scale-up issues. Compared to conventional reactor systems, a short contact time reactor offers a promising alternative-small reactors with high throughput due to fast kinetics. With proper design, quenching of the reacting species in short contact time reactors can also result in higher yield of the intermediates by increasing the rate of chain initiating reactions compared to chain propagation and branching steps. Additionally short contact time reactors can be much smaller thus lowering capital costs. Currently there is a limited understanding of the fundamental processes occurring in short contact time reactors, and this is deterring technological advancement. We have the capabilities, facilities, and expertise to take this concept to the level of fundamental understanding required to make it a truly viable industrial technology.

\section{Importance to LANL's Science and Technology Base and National R\&D Needs}

Theory, modeling, and high-performance computing represent the Laboratory's ability to identify, define, and solve difficult and complex technical challenges by combining fundamental theory with the power of high-performance computing to model a broad range of complicated biological, chemical, and physical processes. In this project we aspire to enhance this core Laboratory competency with a combination of experiments and computer simulations aimed at elucidating the complex chemical and physical processes governing the performance of short contact time chemical reactors.

One aspect of the Laboratory's central mission of reducing the global nuclear danger involves mating the core competency goals with industrial partnerships. As a direct result of the success of this project, we have entered into an industrial collaboration with Dow Chemical, funded by the DOE Office of Industrial Technology. This collaboration is intended to bring light alkane conversion technology to a level of understanding such that industrial scale plants can be built. 
The Department of Energy's energy security mission entails working to assure clean, affordable, and dependable supplies of energy for our nation, now and in the future. To do this requires that we increase the diversity of energy and fuel choices and sources. This project supports that mission through the development of technology the will enable the use of natural gas as a more effective source for fuels and chemicals than is now available.

Another potential national energy mission for which this project is ideally suited is the DOE Office of Fossil Energy's Vision 21 program. This anticipated program is aimed at developing the technologies required to design and build $21^{\text {st }}$ century power plants capable of utilizing a wide range of feedstocks, and producing not only clean electrical power, but a variety of co-produced fuels and chemicals. A short contact time reactor converting natural gas to hydrogen for fuels cells, and synthesis gas for subsequent chemicals production is very well suited for this mission.

\section{Scientific Approach and Accomplishments}

We have used experimentation and modeling together to elucidate some of the important aspects of the chemical and fluid dynamic behavior of short contact time reactors. Gas chromatography diagnostics have been installed for use downstream of the reaction zone to detect and quantify reaction products. We have used computational fluid dynamics (CFD) to provide detailed and spatially resolved information on the fluid and chemical dynamics.

\section{Experiments}

A schematic of the flow reactor components already built, or scheduled to be built, for this project is shown in Figure 1. It consists of: a quartz flow tube with a preheater section; a mesh or ceramic foam catalyst (RCR; optical ports for probing of the flow; a down stream gas sampling probe connected to a gas chromatograph and quadrupole mass spectrometer; flow and temperature controls, and off axis micrometer pinhole TOFMS for monitoring radical and ion species produced at various positions with the reactive flow. The catalyst gauze or monolith is mounted on a coaxial interior flow tube, which can be mated to a micropositioning $\mathrm{X}-\mathrm{Y}-\mathrm{Z}$ translation stage to allow sampling of reactive flows at various positions in the flow reactor geometry. If needed a moveable skimmer cone can be designed to fit within the downstream flow path to sample radial flow patterns. Optical pyrometry, Schlieren imaging, laser-induced-fluorescence (LIF) spectroscopy can also be used to measure temperature, concentration, and flow profiles in reaction volumes at specific positions following the catalytic surface. Optical pyrometry allows for 
measurement of the catalyst surface temperatures. Schlieren imaging can be used to map out flow patterns where necessary. LIF spectroscopy provides similar information, is more sensitive, but less general. The equipment needed to implement these techniques is available and already has been used to make similar measurements in flow reactors.

To date we have succeeded in operating our experimental facility in completely autothermal mode, and achieved the production of ethylene and synthesis gas from an ethane/air feedstock mixture. This represents the culmination of intense efforts in the laboratory by members of both CST-18 and CST-6, and is a major milestone toward the goals of our ongoing DOE funded work.

\section{Modeling}

A two-dimensional modeling effort is in progress which aims to elucidate the fundamental processes governing the catalytic partial oxidation of methane to synthesis gas and ethane to ethylene on platinum in a short contact time reactor. In conjunction with this effort is a modeling program to predict the catalytic ignition temperatures of methane and ethane on platinum in a stagnation flow. The goal is to develop a coupled gas phase and surface kinetic mechanism, which can explain experimental results in both the partial oxidation and ignition regimes. Only with this type of demonstrated broad applicability in our chemical mechanisms can we hope to fully elucidate the fundamental phenomenon associated with alkane conversion to fuels and chemicals. Several aspects of this modeling work have appeared at international meetings and are being prepared for journal publication [1-3].

The steps involved in the modeling of surface catalyzed reactions can be summarized as follows:

1) Transport of reacting gaseous species to the surface

2) Adsorption of these species to the surface

3) Heterogeneous reaction catalyzed by the surface

4) Desorption of gaseous species from the surface

5) Transport of radicals and product species away from the surface

6) Homogenous reaction of radicals and other intermediates to form products

In the presence of very fast surface chemistry and relatively slow gas phase adsorption reactions, the overall reaction progress can be controlled by the transport of species in the bulk phase. To describe an accurate model for surface catalyzed gas 
liquefaction, it is imperative that transport of reactants and products to and from the surface by calculated explicitly.

In flowing short contact time reactor systems, boundary layer development and the associated transport processes can be very complex. The transport mechanisms can vary widely even within a given reactor. It has therefore been necessary to pursue a high fidelity numerical modeling approach that can address the transport issues with the required complexity.

Using FLUENT, a commercial CFD package, we have modeled multiple simultaneous chemical reactions, with reactions occurring in the gas phase and/or on the surface. FORTRAN subroutines external to the FLUENT executable code have been written for the purpose of including detailed surface and gas phase chemistry. As provided commercially, FLUENT has limited capability to include these complicated reaction sets and the associated species source terms. The chemical reaction schemes we are employing can be of arbitrary complexity, and involve rate expressions of any mathematical form. The reactor is modeled with two-dimensional axisymmetric flow equations with full heat and mass transport. The mass fractions of all species in the reaction scheme are tracked with distinct conservation equations. These species conservation equations account for all transport phenomena, including convection, diffusion, and sources and sinks due to chemical reaction. We run FLUENT on a Silicon Graphics Indigo II workstation with an R10000 processor and Solid Impact graphics. Solution times for full steady-state convergence range from several hours to overnight.

Past development of surface kinetic mechanisms for catalytic alkane conversion in short contact time reactors $[4,7]$ has been limited to one-dimensional numerical treatments such as plug flow reactor models. The kinetic mechanisms proposed and the rate parameters derived from this approach incorporate the systematic error that the plug flow assumption represents. An application of the same mechanisms and rate parameters in a two-dimensional treatment with full heat and mass transport fails to predict the experimental data. Complicating matters further is the interaction of gas phase and surface reactions, which is thought to play a much larger role in the ethane to ethylene conversion process than in the methane to synthesis gas process.

We have developed a more complete kinetic mechanism that includes both gas phase and surface reactions. This mechanism has been tested in two-dimensional flow simulations and compared to the experimental data for partial oxidation of methane to synthesis gas. This mechanism, or a subsequent revision, is expected to provide broad applicability so that reliable simulations can be achieved for a wider range of experimental or industrial configurations. To this end, stagnation flow modeling is also to be performed 
to predict the ignition temperatures of ethane and methane in the catalytic combustion regime. This represents a significant extension of previous work in stagnation flow modeling of catalytic combustion processes [8,9], and partial oxidation modeling of the methane to synthesis gas [10] and the ethane to ethylene conversion processes [5].

In Figure 3 are shown results from two-dimensional flow simulations of the partial oxidation of methane to synthesis gas. The results are for a platinum loaded alumina foam monolith. These simulations incorporate only a surface kinetic mechanism. The agreement between the simulation results and two sets of experimental data is quite promising. In Figure 4 are shown the spatial variations along the reactor centerline of species and temperature. Note the sharp gradients that lead to the diffusion of both heat and mass into the region preceding the catalytic wall section.

We have recently demonstrated that gas phase reactions will indeed play an important role in the conversion of methane to syngas. Simulations using a gas phase mechanism reduced to 22 species and 77 reversible reactions show a marked decrease in the selectivities of both $\mathrm{CO}$ and $\mathrm{H}_{2}$. This indicates that further work is yet required so that simulations using a fully coupled chemical mechanism remain in good agreement with the experimental data.

We believe the results of this project have provided a new fundamental understanding of the fluid dynamics and chemical reaction mechanisms driving the conversions and selectivity for short contact time reactors. Experiments performed on these systems and benchmarked modeling which describes both the chemistry and the transport mechanisms have provided insight into the behavior and performance of short contact time reactor designs and will be used to evaluate the economic viability of a full scale reactor system. Successes of this project have provided enhanced Laboratory capabilities in chemical reaction modeling. In addition a new arena for program development and industrial partnership has been opened. This project has led us to a system that is of high value and interest to the DOE and industrial chemical companies. 


\section{Publications}

1. Zerkle, D.K., Deutschmann, O., Paffett, M.T., and Schmidt, L.D., "TwoDimensional Modeling of the Partial Oxidation of Ethane to Ethylene," poster presentation, $27^{\text {th }}$ Symposium (International) on Combustion, Boulder, CO. (1998).

2. Zerkle, D.K., Allendorf, M.D., Deutschmann, O., "Modeling of Catalytic Partial Oxidation and Ignition Processes on Pt with Coupled Gas-Phase and Surface Kinetic Mechanisms", for oral presentation, $16^{\text {th }}$ North American Meeting of the Catalysis Society, Boston, MA, (1999).

3. Zerkle, D.K., Tummala, S., Allendorf, M.D., Deutschmann, O., "Unified Hydrogen Oxidation Kinetics on Platinum", in preparation (1999).

\section{References}

[4] Hickman, D.A., Schmidt, L.D.,J. Catalysis, 138, p. 267 (1992).

[5] Goetsch, D.A.and Schmidt, L.D., Science, 271, pp. 1560-1562 (1996).

[6] Witt, P.M. and Schmidt, L.D., J. Catal., 163, pp. $465-475$ (1996).

[7] Huff, M.C. and Schmidt, L. D., AIChE J., 42, pp. 3484-3497 (1996).

[8] Deutschmann,O., Schmidt, R., Behrendt,F., and Warnatz,J., 26 Symp. (Int'l.) on Comb., p. 1747 (1996).

[9] Warnatz,J., Allendorf, M.D., Kee, R.J., and Coltrin, M.E., Combustion and Flame, 96, p. 393 (1994).

[10] Deutschmann, O., and Schmidt, L.D., AIChE Journal, in press.

[11] Bodke, A., University of Minnesota, Dept. of Chem. Engr. And Matl. Sci., private communication. 
Paffett, 98559, Methane Conversion

Figure 1

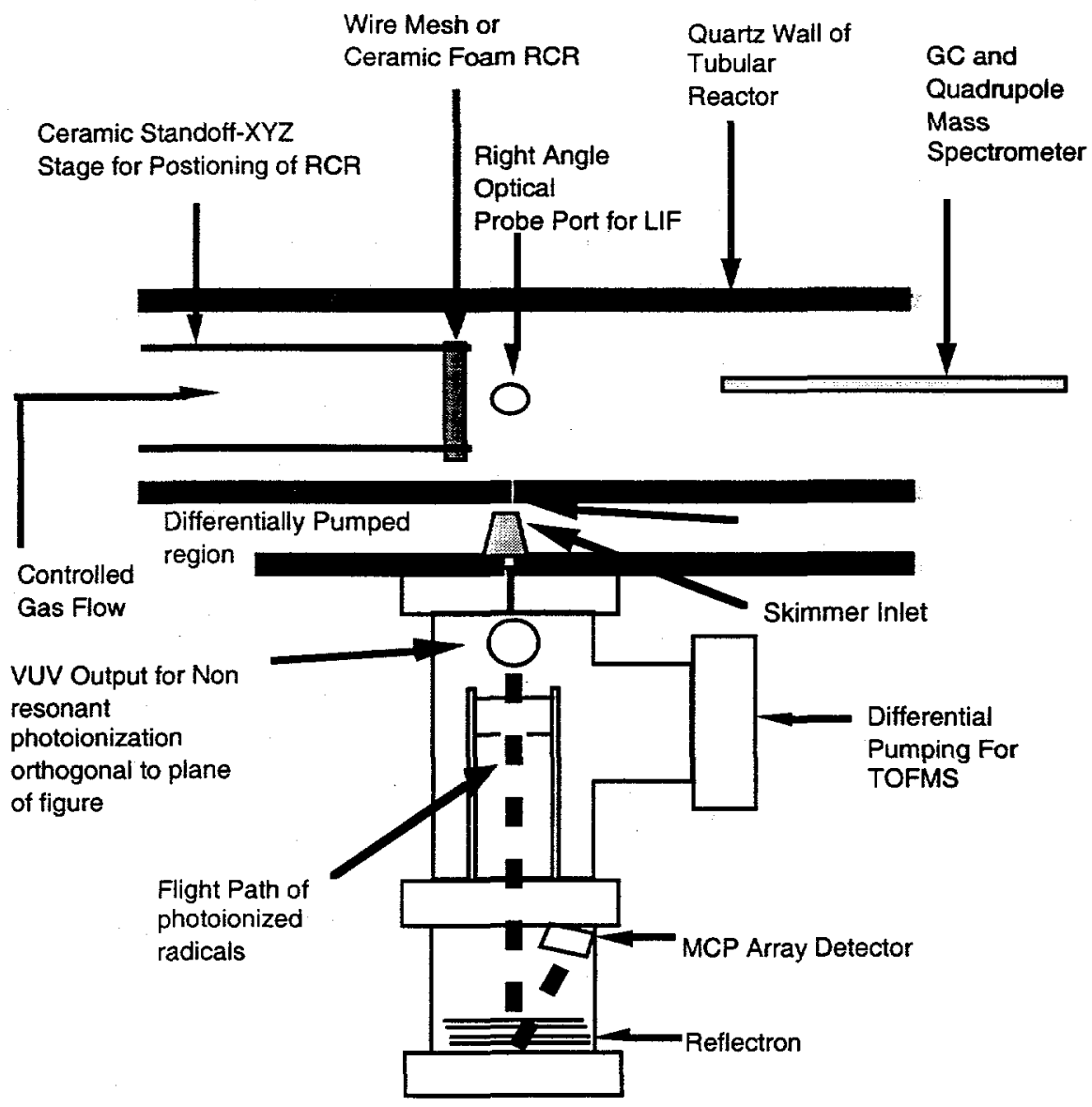

Figure 1 Schematic of experimental short contact reactor (Drawing not to scale). 
Paffett, 98559, Methane Conversion

Figure 2

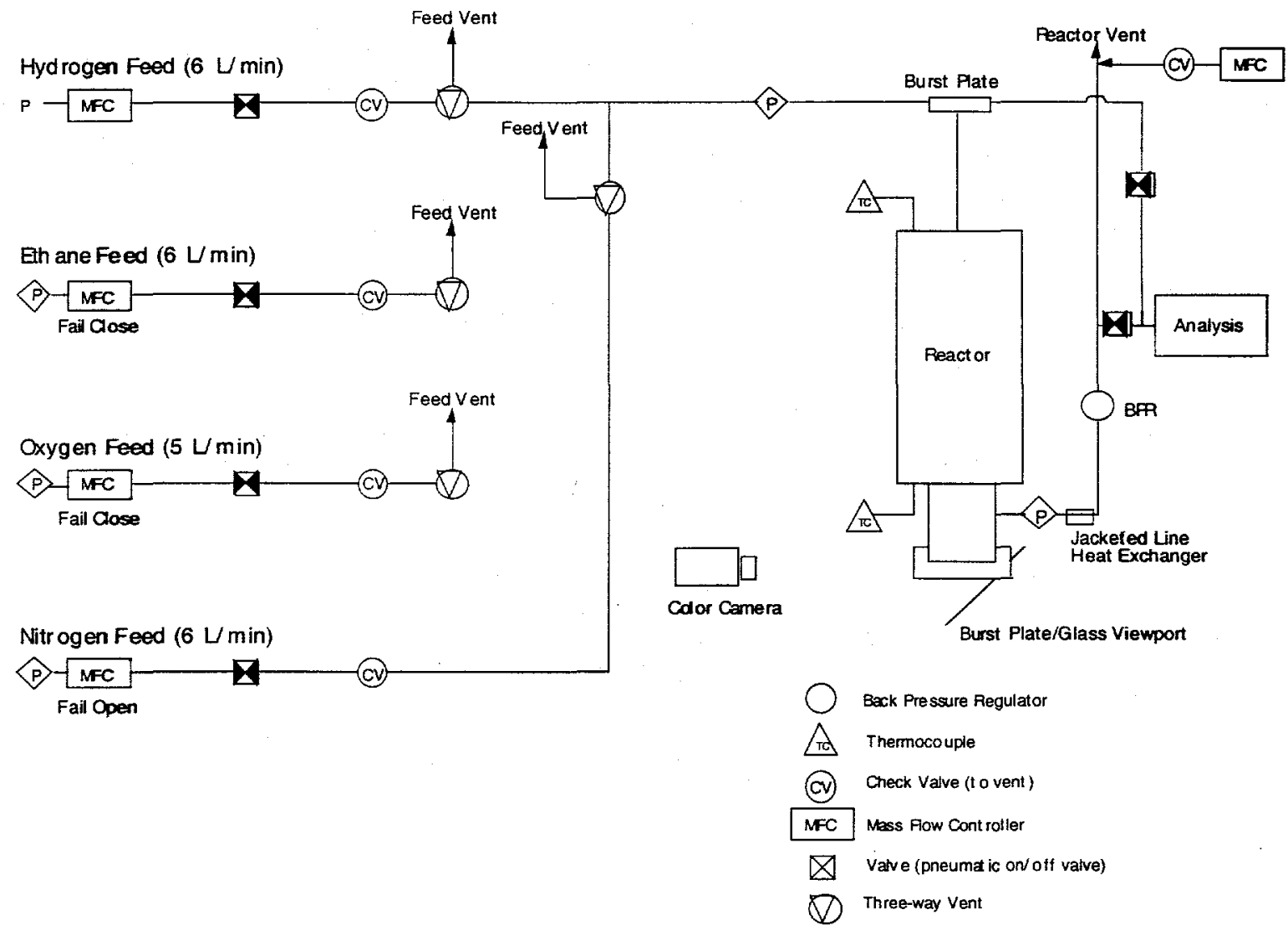

Figure 2 Schematic of overall experimental short contact reactor facility showing flow controls. 
Paffett, 98559, Methane Conversion

Figure 3
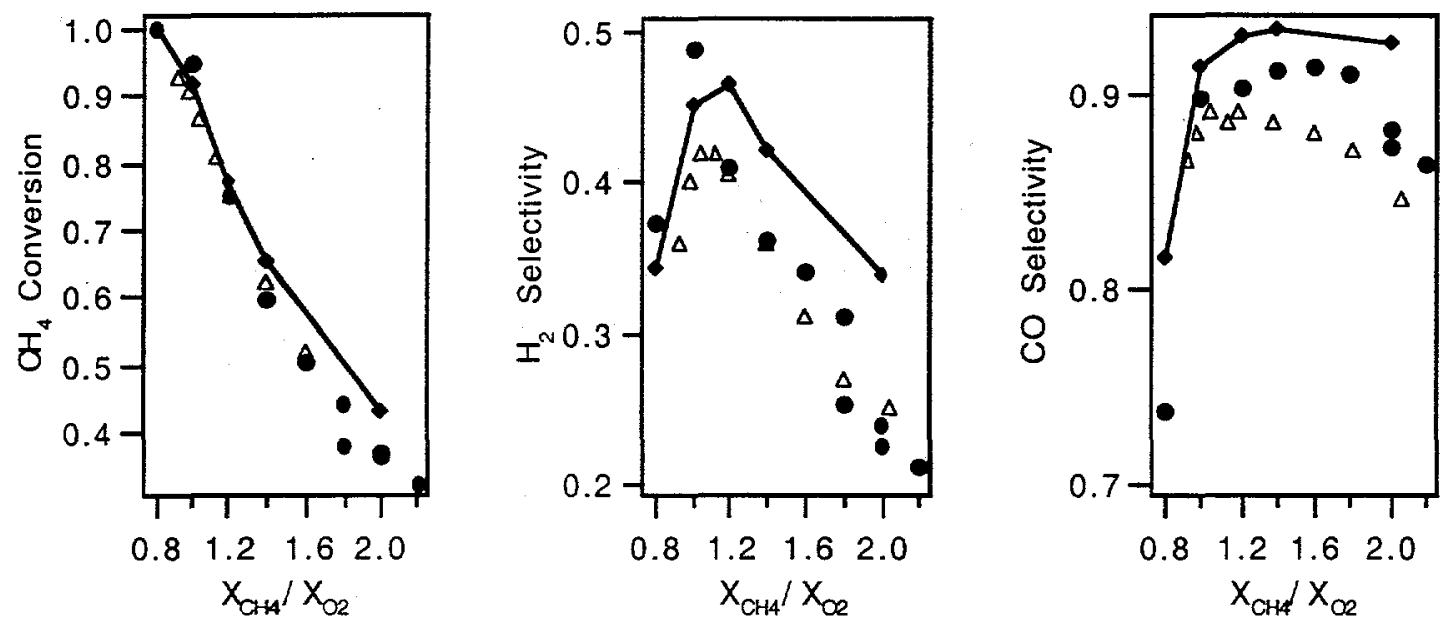

Figure 3 Comparison between calculations and experimental data for methane conversion and synthesis gas selectivities. Current simulation results: connected, closed diamonds $(--)$; Hickman data [1]: open triangles $(\Delta)$; Bodke data [9]: closed circles $(\bullet)$. 
Paffett, 98559, Methane Conversion

Figure 4

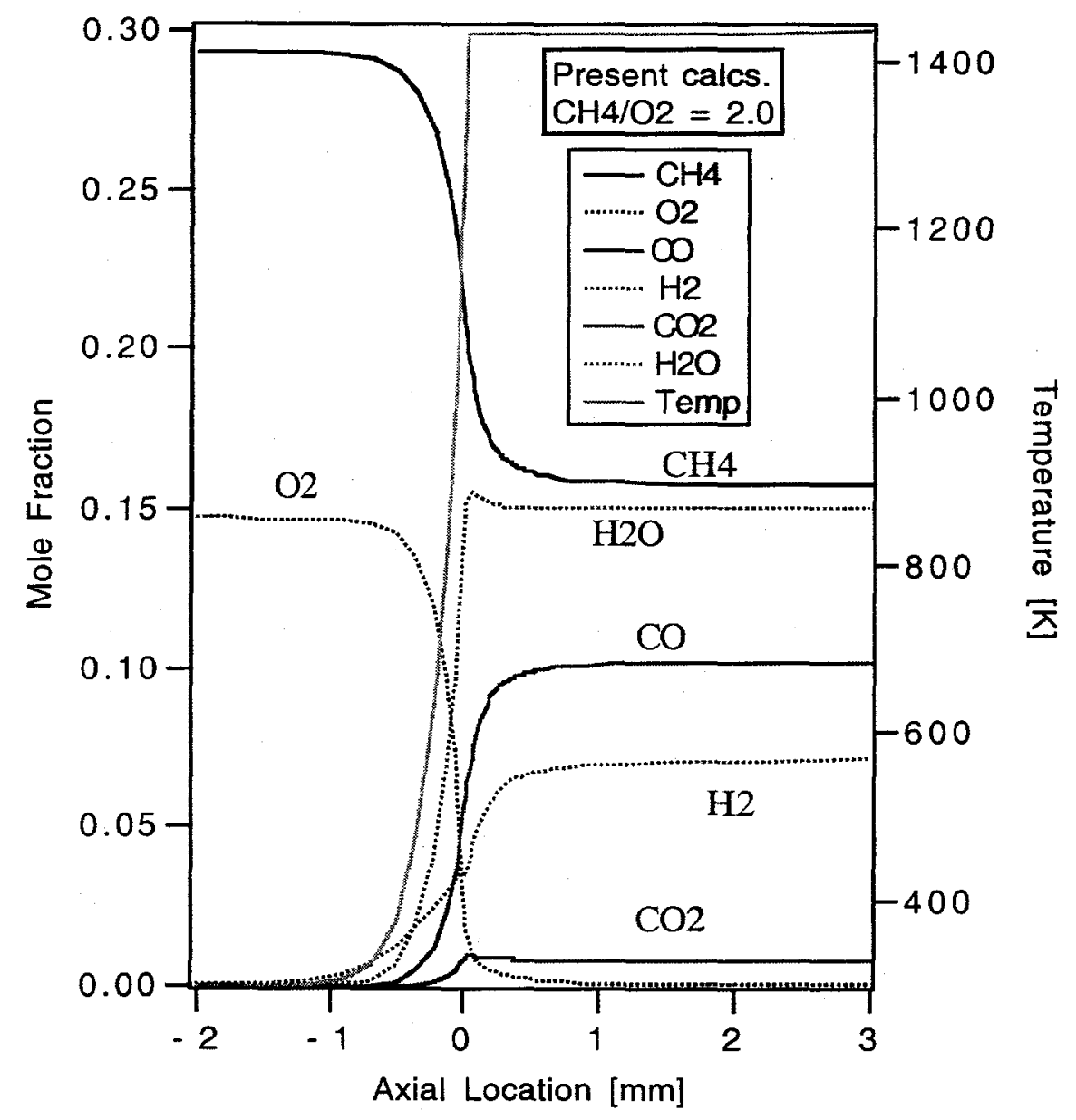

Figure 4 Computer simulation of the axial variation of species and temperature near the start of the catalytic wall section in a short contact time reactor. 